\title{
LONGITUDINAL IMPEDANCE TUNER USING HIGH PERMEABILITY MATERIAL
}

\author{
$\underline{\text { K.Koba }}^{1}$, D.Arakawa, M.Fujieda, K.Ikegami, Y.Ishi, Y.Kanai, C.Kubota, S.Machida, Y.Mori, \\ C.Ohmori, K.Shinto, S.Shibuya, A.Takagi, T.Toyama, T.Uesugi, T.Watanabe, M.Yamamoto, \\ T.Yokoi, and M.Yoshii, KEK, Tsukuba, Japan
}

\begin{abstract}
We have succeeded in canceling longitudinal space charge effects using the 'impedance tuner' [1][2][3] at the KEK-PS. The impedance tuner consists of the new material, 'FINEMET' [4][5][6][7] which has large permeability over the beam spectrum region. A frequency shift of coherent quadrupole mode is measured to infer the modified impedance. It turns out that nonlinearity unavoidable in a longitudinal rf bucket has to be treated carefully in order to digest observed beam signals. We will describe how to measure the impedance from the beam signal, how to analyze the effect of impedance tuner, and how to succeed in canceling space charge effects.
\end{abstract}

\section{INTRODUCTION}

In a high intensity proton synchrotron, some of the emittance growth and beam instabilities are caused by space charge effects. In the longitudinal phase space, the space charge forces weaken the $\mathrm{rf}$ focusing force. When a short bunch is required, for example in a proton driver of a muon collider, the effects are further enhanced and they limit the minimum bunch length.

An inductive device in a ring should be able to cancel the space charge force. Recently a very high permeability material, FINEMET, becomes available. It turns out that the material has enough permeability at the beam frequency region and possibly cancels the space charge impedance. We designed a device, and named it "impedance tuner". It consists of FINEMET cores and is installed in the KEK PS main ring.

\section{ESTIMATE OF IMPEDANCE}

The space charge impedance is negative inductance and written as,

$$
\frac{Z_{s c}}{n}=-j \frac{g_{0} Z_{0}}{2 \beta \gamma^{2}},
$$

where $Z_{0}$ is the free space impedance, $\beta$ and $\gamma$ are Lorenz factors, and $g_{0}$ is a form factor defined by

$$
g_{0}=1+2 \ln \frac{a}{b} \text {. }
$$

Here $a$ is the radius of beam pipe and $b$ is the transverse beam radius.

On the other hand, FINEMET has positive inductive impedance. The inductive impedance is expressed as

$$
\frac{Z_{\text {ind }}}{n}=j \omega_{0} L,
$$

where $\omega_{0}=2 \pi f_{0}$ and $f_{0}$ is the revolution frequency (667

${ }^{1}$ koba@psacw01.kek.jp
$\mathrm{kHz}$ ), and $L$ is the inductance at that frequency.

The impedance tuner is installed in the KEK PS main ring. Let us first calculate the space charge impedance. Since the size of beam pipe is about $150 \mathrm{~mm}$ and the average beam size is about $60 \mathrm{~mm}$, the form factor $g_{0}$ becomes 2.8 . Together with the other parameters of a $500 \mathrm{MeV}$ beam, the space charge impedance becomes $-j 310 \Omega$.

In order to observe space charge effects and its compensation with the impedance tuner, an experiment was carried out using beams at the injection energy.

We take the revolution frequency and characterize the impedance by it because we observe the effects of the impedance tuner on a single bunch whose fundamental frequency is the revolution. In order to cancel space charge impedance of $-j 310 \Omega$ completely, the total inductance of $73.7 \mu \mathrm{H}$ should be prepared with FINEMET.

The FINEMET is wound to a toroidal core with the outer diameters of $340 \mathrm{~mm}$, the inner diameter of $140 \mathrm{~mm}$, and the thickness of $25 \mathrm{~mm}$. We measured the inductance of FINEMET as a function of frequency is inductive impedance is about $j 25.5 \Omega$ at the injection revolution frequency. Therefore, the total of 12 pieces of FINEMET core are necessary to fully compensate space charge impedance.

\section{EXPERIMENTAL SET UP}

The impedance tuner consists of identical three units and each unit has 4 pieces of FINEMET core, ceramic gaps and cooper shields as shown in Fig.1. All of the FINEMET cores are placed outside of the ceramic gaps. There are short to observe the difference between with and without the short bars. The total length is $1.2 \mathrm{~m}$.

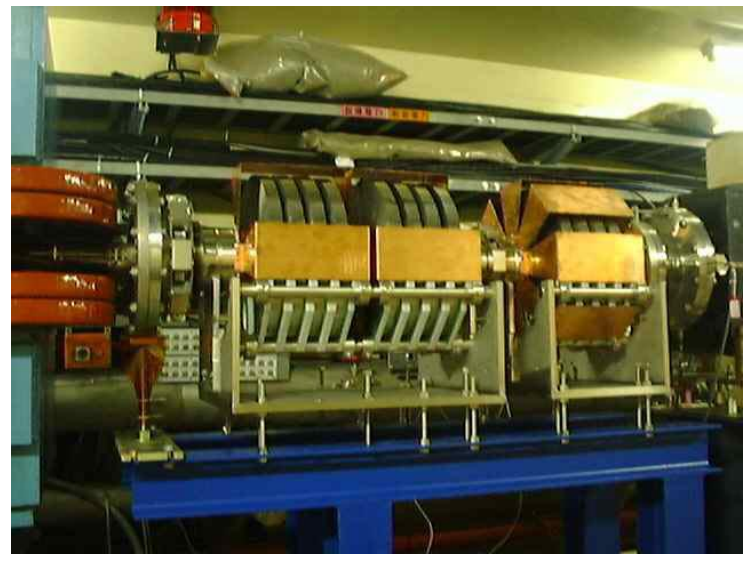

Fig.1 Impedance tuner installed in the KEK PS. 


\section{RADIATION EFFECT}

This is the first time to apply FINEMET to an accelerator. It may happen that radiation due to beam loss causes a deterioration in the characteristic of FINEMET. We have proved that the radiation dose not affect the impedance of FINEMET with the following procedure. A small sample of FINEMET was placed for three weeks at the section of the beam extraction where the radio activation becomes maximum in the main ring. Then the impedance was measured with a network analyzer. The same procedure was repeated every three weeks.

Figure 2 show the imaginary part of the impedance. For nine week, the total neutron flux applied to FINEMET was $1.83 \times 10^{12}\left[\mathrm{n} \cdot \mathrm{cm}^{-2}\right]$. We could conclude that the impedance of the FINEMET was not changed by the total neutron flux of $1.83 \times 10^{12}\left[\mathrm{n} \cdot \mathrm{cm}^{-2}\right]$. The impedance tuner were installed in the place where the beam loss was much less than the beam extraction section.

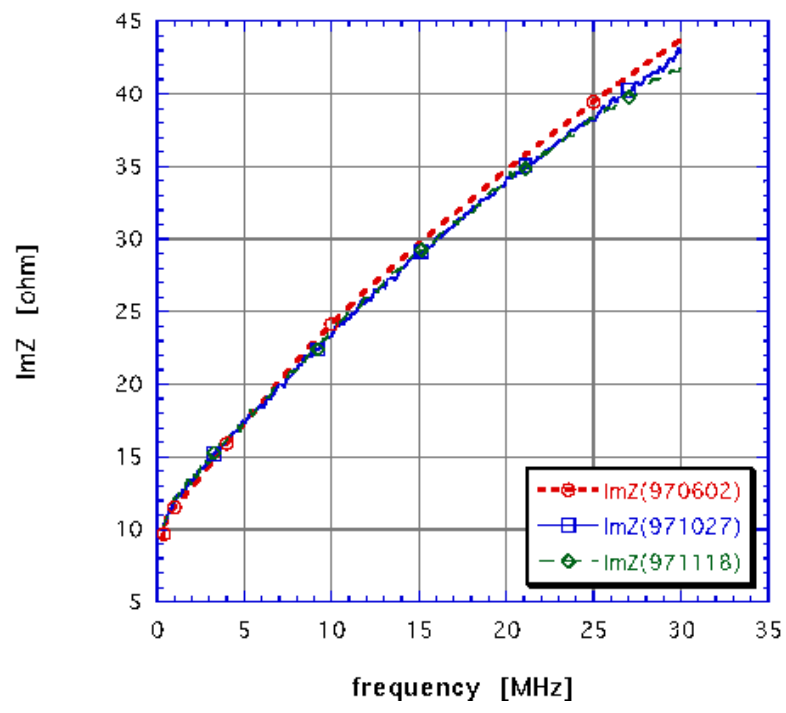

Fig.2 Imaginary impedance of the FINEMET.

\section{MEASUREMENT}

The frequency $f_{s 0}$ of synchrotron oscillations is perturbed with the potential of the space charge and the inductive impedance. We will measure the shift of synchrotron frequency $\Delta f_{s}$ as a function of beam intensity and obtain total impedance as a coefficient.[8]

In fact, the shift of incoherent synchrotron oscillations can not be measured directly. However, the incoherent frequency shift can be inferred from the coherent quadrupole oscillations. If we take $\Delta f_{s 2}$ as a frequency shift of the quadrupole oscillations, there is the following relation with the incoherent shift[9].

$$
\frac{\Delta f_{s 2}}{f_{s 20}} \approx \frac{1}{4} \frac{\Delta f_{s}}{f_{s 0}}
$$

where $f_{s 20}$ is the coherent quadrupole frequency at zero beam intensity.
The frequency of the quadrupole oscillations is measured as the envelope oscillations of a bunch signal from a wall current monitor.

The frequency of the quadrupole oscillations was measured as a function of beam intensity using impedance tuner and shown in Fig.3. The solid line is a fitted line of the data when all of the ceramic gap were shorted. The dashed line is the one when one ceramic gap was shorted and the rest of the gaps were opened. The long-dashed line is the one when all of the gaps were opened.

We estimate the non-perturbed quadrupole frequency $f_{s 20}$ by extrapolating the measured frequency to that of zero beam intensity, and list the results on the Table1. The average synchrotron frequency is $10.27 \mathrm{kHz}$. The reactive impedance on each condition is derived from the slope using the average synchrotron frequency and listed in Table2. As expected, the more inductance is installed, the lower magnitude of impedance is obtained. That demonstrates the space charge impedance is compensated with the impedance tuner.

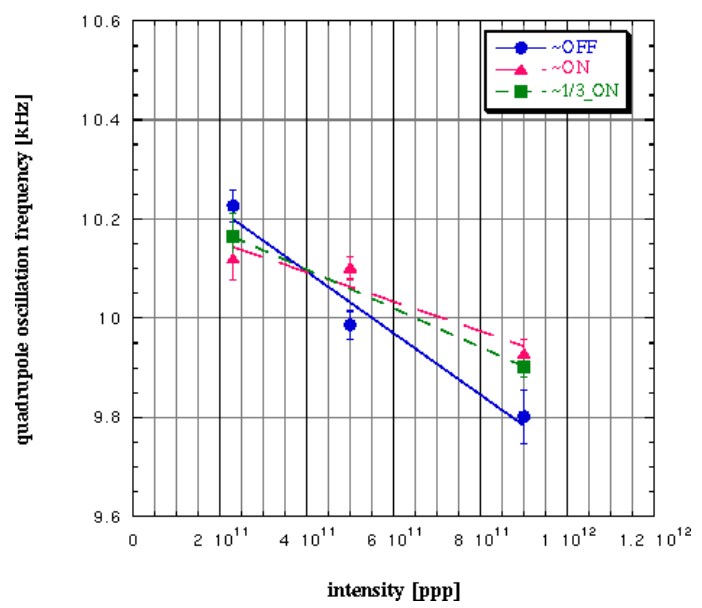

Fig.3 Quadrupole oscillation frequency estimated from the bunch envelope measurement.

Table1: synchrotron frequency ( at zero intensity)

condition $f_{0}[\mathrm{kHz}]$

without cores $\quad 10.34$

with $1 / 3$ of total cores $\quad 10.25$

with total cores $\quad 10.21$

Table2: Reactive impedance on each condition is derived from the slope.

$\begin{array}{ll}\text { condition } & \text { reactive impedance }[\Omega] \\ \text { without cores } & -j 2475 \\ \text { with } 1 / 3 \text { of total cores } & -j 1554 \\ \text { with total cores } & -j 1182\end{array}$

\section{EFFECT OF THE RF NONLINEAR FIELD}

We have observed that the measured space charge impedance was reduced from $-j 2475 \Omega$ to $-j 1182 \Omega$ by the impedance tuner which consisted of 12 pieces of the 
FINEMET cores. However, the measured impedance of $-j 2475 \Omega$ was 8 times as large as the impedance of $-j 310 \Omega$ obtained from the calculation. Moreover, the quadrupole frequency at the zero beam intensity was lower than the frequency, $12.44 \mathrm{kHz}$, estimated by the following equation (5) and Table 3.

$$
f_{2 s 0}=2 \times f_{s 0}=2 \times f_{r e v} \sqrt{\frac{-\eta h}{2 \pi \beta^{2} \varepsilon_{s}} e V_{0} \cos \phi_{s}} .
$$

Table 3: Nominal values of the KEK PS main ring at injection.

symbol

$\begin{array}{ll}R ; \text { machine average radius } & 54 \mathrm{~m} \\ \eta ; \text { slippage factor } & -0.42 \\ V_{0} ; \text { RF voltage } & 120 \mathrm{kV} \\ h ; \text { harmonic number } & 9 \\ \phi_{s} ; \text { synchronous phase } & 0 \mathrm{deg} \\ f_{\text {rev }} ; \text { revolution frequency } & 667 \mathrm{kHz}\end{array}$

These two inconsistencies were causes by the nonlinearity of the RF field. The equation (4) and (5) are based on the assumption that an RF field is linear. The eq.(4) also assumes that the particles distribution matches to the RF bucket shape. However, the bunch occupies the most of the RF bucket area and not matches to it under the operational condition of the KEK PS main ruing. We have examined the difference between linear and non-linear effects by the multiparticle simulation. The results of the simulation are shown as follows.

\subsection{Simulation with linear field}

Assuming a linear field without space charge (zero intensity), the frequency of the envelope oscillation (the coherent quadrupole frequency $f_{s 20}$ ) is twice frequency of the single particle motion in the bunch (incoherent frequency $\left.f_{s 0}\right)$, which is $12.46 \mathrm{kHz}$.

Assuming a linear field with space charge, the relation of the frequency shift agree with the eq.(4).

\subsection{Simulation with non-linear field}

It turns out that assuming the non-linear field without space charge, the envelope oscillation frequency $f_{s 20}$ dose not agree with that estimated by eq.(5). We found that the frequency obtained by the envelope oscillation becomes an average frequency $\left\langle f_{s 0}\right\rangle$, which is $10.60 \mathrm{kHz}$, because each particle has each incoherent synchrotron frequency as a function of the amplitude. This value is consistent with the result of the measurement.

Assuming a non-linear field with space charge, the frequency shift is written by eq.(6) rather than (4)

$$
\frac{\Delta f_{s 2}}{f_{s 20}} \approx \frac{1}{2} \frac{\Delta f_{s}}{f_{s 0}} \text {. }
$$

As a result of these examinations, we know that if the bunch is influenced by the RF nonlinear field strongly, the impedance has been over estimated.

We have tried again the impedance analysis considering the RF non-linear effect. As a result, the impedance became $-j 697 \Omega$ and was closed to the result of the calculation as shown in Fig.4. The detail has been discussed in ref.[10].

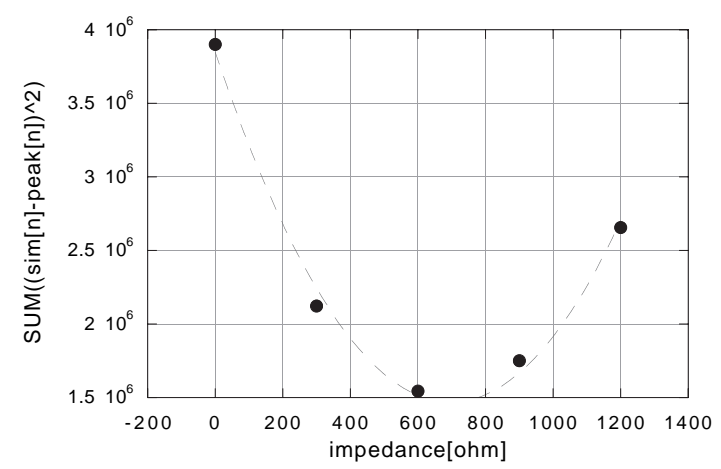

Fig.4 Analyzed impedance including RF non-linearlity.

\section{SUMMARY}

We designed the impedance tuner consisting of the inductive material, FINEMET, to cancel the space charge impedance in the longitudinal direction. It was installed in the KEK PS main ring.

We observed the frequency shift of the coherent quadrupole oscillations and inferred the shift of the incoherent synchrotron oscillation. Total reactive impedance can be estimated as the coefficient between the shift and beam intensity. The measured impedance is reduced from $-j 2475 \Omega$ to $-j 1182 \Omega$ by the impedance tuner which consists of 12 pieces of the FINEMET cores. We demonstrated the space charge impedance is compensated by the impedance tuner.

This is the first time for FINEMET to be equipped with as an accelerator component. We have proved that the radiation dose not affect the FINEMET even with the total neutron dose of $1.83 \times 10^{12}\left[\mathrm{n} \cdot \mathrm{cm}^{-2}\right]$, which is considered as the highest dose of the main ring.

We have analyzed the RF non-linear effect in detail with calculation and simulation.

\section{REFERENCES}

[1]K.Koba et al., Proceedings of the 11th Symposium on Accelerator Technology and Science, 1997, pp.409-411.

[2]K.Koba et al, Proceedings of the APAC,1998.

[3]K.Koba et al, RSI, to be published.

[4]T.Uesugi et al., JHF internal report, JHP-31, 1997.

[5]Y.Yoshizawa et al., Journal of Applied Physics, vol.64, 1988, pp.6044-6046.

[6]Y.Yoshizawa et al., IEEE, vol.25, No5, 1989, pp.3324-3326.

[7]Y.Mori et al., Proceedings of the EPAC,1998, pp.299-301.

[8]S.Hansen, IEEE vol.NS-22, NO.3, 1975.

[9]F.Sacherer, CERN/SI-BR/72-5, 1972.

[10]K.Koba, doctor thesis, 1999. 